\title{
A Psicóloga no Contexto dos Cuidados Paliativos: Principais Desafios
}

\author{
The Psychologist in the Context of Palliative \\ Care: Main Challenges
}

\section{Rafaela Novis Edington ${ }^{1}$ Carolina Villa Nova Aguiar ${ }^{2}$ Eliana Edington da Costa e Silva ${ }^{3}$ (c)}

${ }^{1}$ Autora para correspondência. Escola Bahiana de Medicina e Saúde Pública (Salvador). Bahia, Brasil. rafaelaedington16.1@bahiana.edu.br ${ }^{2-3}$ Escola Bahiana de Medicina e Saúde Pública (Salvador). Bahia, Brasil. carolinaaguiar@bahiana.edu.br, eecesilva@bahiana.edu.br

RESUMO | INTRODUÇÃO: Devido aos avanços tecnológicos, a morte passou a ser vista pelos profissionais de saúde como um fracasso profissional. O campo de cuidados paliativos é recente e visa cuidar de pacientes de forma integral, promovendo a melhoria da qualidade de vida e o alívio da dor diante de uma doença que ameace a vida. O psicólogo se faz importante nessa equipe multiprofissional por acolher o sofrimento psíquico dos pacientes e familiares diante da morte e luto. OBJETIVO: Identificar os principais desafios percebidos por psicólogas(os) que atuam no contexto dos cuidados paliativos em Salvador/BA. MÉTODO: Trata-se de uma pesquisa descritiva de caráter qualitativo e corte transversal, que contou com a participação de 8 psicólogas. O instrumento utilizado foi um questionário online que constava de uma ficha de dados sociodemográficos e ocupacionais e questões abertas. Foi utilizada a análise de conteúdo de Bardin. RESULTADOS: Foi possível identificar quatro categorias: "comunicação e atuação com a equipe multiprofissional", "atendimento à pacientes e familiares", "condições de trabalho" e "falta de reconhecimento profissional" - sendo a primeira categoria a que obteve maior destaque. CONCLUSÃO: A psicologia hospitalar se trata de um campo recente e em consolidação, por isso, ainda é difícil o entendimento da equipe e gestão acerca da função da psicóloga. Dessa forma, é necessário uma melhor estruturação e comunicação em relação ao fazer deste profissional no contexto de cuidados paliativos, bem como maior preparo nos cursos de graduação e especialização para lidar com temas relacionados a finitude.

PALAVRAS-CHAVE: Cuidados Paliativos. Psicologia. Psicologia Hospitalar.

\begin{abstract}
INTRODUCTION: After significant technological improvements in medicine, death started to be seen by health professionals as a professional failure. The field of palliative care is recent and aims to take care of patients in an integral way, promoting life's quality improvement and pain relief regarding a potentially fatal disease. The psychologist is important in this multi-professional team for welcoming the psychological suffering of patients and family members about death and grief. OBJECTIVE: Identify the main perceived challenges from psychology professionals who work in the context of palliative care in Salvador/BA. METHOD: This is a qualitative and cross-sectional descriptive research was carried out with the participation of 8 psychologists. The method applied was an online questionnaire that consisted of a sociodemographic and occupational datasheet and open questions. The Bardin content analysis was chosen as the main framework. RESULTS: It was possible to identify four categories: "communication and performance with the multidisciplinary team," "care assistance for patients and family members," "working conditions," and "lack of professional recognition"; The first category stood out the most. CONCLUSION: Hospital psychology is a recent and consolidating field, so it is still difficult for the team and management to understand the psychologist's role. Finally, it is desired to design a better planning and communication approach concerning psychology professionals' role in palliative care. Also, it is relevant even more studies in undergraduate and specialization courses related to finitude.
\end{abstract}

KEYWORDS: Palliative Care. Psychology. Hospital Psychology. 


\section{Introdução}

O tema morte ainda é tratado como um tabu em muitos ambientes no contexto contemporâneo (Santos \& Pintarelli, 2019). Na cultura brasileira, a ideia de morte está constantemente associada a sentimentos negativos como dor, separação, perda e sofrimento; e, ao lidar com a partida do outro, as pessoas percebem a sua própria finitude (Perboni et al., 2018).

Comparando o cenário atual com a Idade Média, pode-se dizer que o homem "desaprendeu" a conviver com a fase final da vida, que antes era enfrentada como um processo mais natural (Perboni et al., 2018). Em grande medida, é possível atribuir essa mudança aos avanços na área da medicina, intensificados a partir do século XX, que resultaram em uma elevação da expectativa de vida tão expressiva a ponto de inverter a pirâmide populacional, em que o subgrupo de idade entre 80 e 89 anos é o que mais cresce na sociedade moderna (Gomes \& Othero, 2016).

As inúmeras possibilidades oferecidas pelo avanço tecnológico, como os novos exames, intervenções e tratamentos, refletiu, inevitavelmente, na própria formação e atuação dos profissionais de saúde: a ênfase, cada vez mais, passou a recair sobre uma perspectiva curativa. A grande preocupação está atrelada à cura e tratamento ativo da doença que, muitas vezes, deixa de lado questões como a perda da qualidade de vida e o grau de sofrimento gerado para o indivíduo e seus familiares. A morte deixa de ser vista pelos profissionais de saúde como um processo natural e necessário e passa a ser encarada como uma representação do seu fracasso e impotência (Silveira et al., 2014). De acordo com Alves, Cunha et al. (2019), ainda hoje a graduação em medicina não ensina como lidar com a morte de forma humanizada e ativa.

Apesar de essa ser a situação predominante, pode-se dizer que estamos vivendo um período de transição, que teve início com o surgimento do movimento paliativista. Este se propõe a oferecer uma atenção mais integral, promovendo a melhoria da qualidade de vida do paciente e de seus familiares, diante de uma doença que ameace a vida (Organização Mundial da Saúde [OMS], 2002). E, para a realização desse cuidado, se faz imprescindível profissionais da psicologia como integrantes das equipes que oferecem cuidados paliativos.
Por cuidados paliativos se tratar de um campo de atuação recente e inovador e, especialmente, por estar intrinsecamente associado a um fenômeno ainda carregado de tantos estigmas na sociedade ocidental - a morte - trabalhar no contexto dos cuidados paliativos requer dos profissionais de saúde o constante manejo de fatores potencialmente estressores. E pelos profissionais da psicologia estarem, frequentemente, em contato com esse sofrimento psíquico gerado pelo luto e se tratar, também, de uma atuação recente, este estudo se torna relevante. Diante do exposto, o presente trabalho tem o objetivo de identificar os principais desafios percebidos por profissionais da psicologia que atuam no contexto dos cuidados paliativos na cidade de Salvador/BA.

\section{Cuidados paliativos}

\section{História do movimento paliativista}

O movimento paliativista começou a ganhar forma na Inglaterra, em 1960, com Cicely Saunders, enfermeira, médica, assistente social e fundadora do St. Christopher's Hospice, em 1967 - primeiro serviço a oferecer cuidado integral ao paciente (ANCP, s.d.).

A Organização Mundial da Saúde (OMS, 2002) definiu pela primeira vez o conceito e princípios das práticas paliativas em 1990 e, em seguida, em 2002. A mais recente publicação define como:

"assistência promovida por uma equipe multidisciplinar, que objetiva a melhoria da qualidade de vida do paciente e seus familiares, diante de uma doença que ameace a vida, por meio da prevenção e alívio do sofrimento, da identificação precoce, avaliação impecável e tratamento de dor e demais sintomas físicos, sociais, psicológicos e espirituais".

(OMS, 2002)

Também aborda a prevenção e controle de sintomas, intervenção psicossocial e espiritual, paciente e família como unidade de cuidados, autonomia e independência, e comunicação e trabalho em equipe multiprofissional, como princípios norteadores do trabalho em cuidados paliativos (OMS, 2002).

No Brasil, os serviços organizados começaram na década de 1990 e desde então o movimento vem crescendo. Saltos importantes foram dados em 1997 
e 2005 com a fundação da Associação Brasileira de Cuidados Paliativos (ABCP) e da Academia Nacional de Cuidados Paliativos (ANCP), respectivamente - o que colaborou para a consolidação e regularização da assistência paliativa no país (ANCP, s.d.). Além disso, o Conselho Federal de Medicina (Resolução n. 1.973, 2011) incluiu o Cuidado Paliativo (CP) como área de atuação em 2011, importante reconhecimento conquistado pelo movimento.

Segundo o Atlas de Cuidados Paliativos no Brasil (Santos et al., 2020), registrou-se um crescimento positivo de 7,9\% nos serviços de CP de 2018 para 2019, e em 2019 foram contabilizados o total de 191 serviços de CP no país (798 leitos), com destaque para o estado de São Paulo, com 66. Do total, dos referidos serviços oferecidos no Brasil, $50 \%$ são de atendimento público, 36\% são de iniciativa privada e $14 \%$ atendem pelo SUS e de forma particular. Ainda, 37,2\% dos serviços têm vínculos com cursos de graduação, 19,1\% com cursos de pós-graduação e 57,1\% com programas de residência. Apesar do crescimento, verificase que há, em média, apenas um serviço de CP para cada 1,1 milhão de habitantes, mesmo havendo a recomendação de dois serviços especializados de CP a cada 100.000 habitantes (uma equipe de assistência domiciliar e uma equipe de nível hospitalar).

Sobre a Bahia, o foco do presente estudo, 5,2\% destes serviços se encontram nesse estado. Eles têm, em média, 3,08 anos de funcionamento, o que confirma quão recentes eles são. A rede privada responde por $60 \%$ dos serviços e, apesar de apenas $30 \%$ do total de serviços na Bahia possuírem vínculo com cursos de graduação e $40 \%$ com programas de residência, os serviços no estado têm $100 \%$ de envolvimento em pesquisa (Santos et al., 2020).

\section{A psicologia hospitalar e a atuação em cuidados paliativos}

A Lei $n^{\circ}$ 4.119/1962 (1962) reconhece a profissão de psicólogo, fixa normas para a atuação profissional e estabelece um currículo mínimo para sua formação. No ano de 1971, o Conselho Federal de Psicologia (CFP) e os Conselhos Regionais (CRP) foram criados pela Lei $n^{\circ} \underline{5.766}$ (1977) que só foi regulamentada em 1977, pelo Decreto 79.822 (1971). No ano 2000, a Psicologia Hospitalar foi reconhecida pelo CFP como uma especialidade (Resolução n. 014, 2000).
Em seguida, a resolução $n^{\circ} \underline{013}$ (2007) instituiu a consolidação das resoluções relativas ao Título Profissional de Especialista em Psicologia e dispõe sobre normas e procedimentos para seu registro, incluindo a área hospitalar.

O profissional da psicologia hospitalar atua em instituições de nível secundário ou terciário da atenção à saúde e, também, em instituições de ensino superior e/ou centros de estudo e de pesquisa. Pode desenvolver diferentes modalidades de atuação como, por exemplo, atendimento psicoterapêutico, grupos psicoterapêuticos, grupos de psicoprofilaxia, atendimentos em ambulatório e unidade de terapia intensiva, pronto atendimento, enfermarias em geral, psicomotricidade no contexto hospitalar, avaliação diagnóstica, psicodiagnóstico, consultoria e interconsultoria (Resolução n. 02, 2001).

Referente à prática profissional, a resolução $\mathrm{n}^{\circ}$

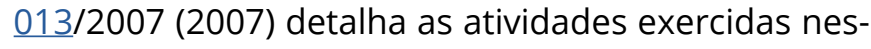
te campo, que são:

a) Atendimento a pacientes, familiares e/ou responsáveis pelo paciente; membros da comunidade dentro de sua área de atuação; membros da equipe multiprofissional e eventualmente administrativa, visando o bem-estar físico e emocional do paciente;

b) Avaliação e acompanhamento de intercorrências psíquicas dos pacientes que estão ou serão submetidos a procedimentos médicos, visando basicamente a promoção e/ou a recuperação da saúde física e mental;

c) Promoção de intervenções direcionadas à relação médico/paciente, paciente/família, paciente/paciente e do paciente em relação ao processo do adoecer, hospitalização e repercussões emocionais que emergem neste processo;

d) Participação na formulação de decisões em relação à conduta a ser adotada pela equipe, aportando informações pertinentes à sua área de atuação;

e) Realização de grupo de reflexão com a equipe de saúde, no qual o suporte e manejo estão voltados para possíveis dificuldades operacionais e/ou subjetivas dos membros. 
No ambiente hospitalar, a psicóloga está inserida em uma equipe multiprofissional, o que torna indispensável a troca de saberes para satisfazer as necessidades globais do paciente. A atuação em equipe pode vir a acontecer de forma fragmentada, prejudicando o desenvolvimento do sujeito que está sob cuidados. $\mathrm{O}$ tratamento recebido dos profissionais e o modo como se relaciona com os mesmos definirão o enfoque do atendimento psicológico, podendo ser facilitadores ou dificultadores no processo (Fossi \& Guareschi, 2004).

No desenvolvimento em equipe multiprofissional, é importante estar definida a função de cada membro e os seus limites, para evitar disputas de poder e problemas na atuação e comunicação. Mas, ainda se faz necessária a discussão e reflexão sobre a assistência prestada no que diz respeito à terminalidade (Cardoso et al., 2013).

A psicóloga que atua no contexto de CP tem como função definida pelo Manual de Cuidados Paliativos da ANCP (Carvalho \& Parsons, 2012) oferecer escuta clínica, facilitar a elaboração das vivências associadas ao adoecimento, processo de morte e luto, além de estimular o doente e família a falarem livremente sobre a sua situação - habilidade de manejo grupal. Segundo Hermes e Lamarca (2013), o principal objetivo deste profissional, nessa área, consiste em atuar nas desordens psíquicas que geram estresse, depressão e sofrimento fornecendo um suporte emocional à família que permita a ela conhecer e compreender o processo da doença nas suas diferentes fases.

\section{Desafios dos profissionais no contexto hospitalar}

O ambiente hospitalar é considerado, por muitos, um espaço de trabalho insalubre por ter que lidar diariamente com pessoas debilitadas, com a dor, com o sofrimento e com a morte, o que o torna um lugar altamente propício a situações estressantes (Silva et al., 2015). Destarte, já existem na literatura estudos com foco nos profissionais de saúde a fim de investigar e cuidar da saúde mental destes, principalmente, por lidarem com pessoas em estado vulnerável, o que necessita que eles estejam aptos para desempenhar essa função (Cardoso et al., 2013; Maturana \& Do Valle, 2014; Silva et al., 2015).
São muitos os desafios enfrentados por uma equipe multidisciplinar no contexto hospitalar. Os desafios divergem dependendo do indivíduo, da situação em que se encontra, e da função que exerce. Em relação à Psicologia, ainda são poucos os estudos referentes aos desafios e estresses ocupacionais vivenciados por esses profissionais. Devido à carência de disciplinas que tratem da morte na graduação, como apontado pela pesquisa de Hermes e Lamarca (2013), iniciar a prática em cuidados paliativos deve ser trabalhoso e estressante. Segundo Maturana e Do Valle (2014), para lidar com aspectos relacionados à terminalidade, a psicóloga depara-se com situações que impõem controle e administração de emoções a fim de amenizar o sofrimento sentido pela perda. A carga social em relação ao tema, o intenso sofrimento gerado pelo adoecimento e as diferenças entre a equipe na forma de lidar com os desafios são fatores potencialmente estressores para a psicóloga.

\section{Metodologia}

Trata-se de uma pesquisa descritiva, de abordagem qualitativa e corte transversal. Para responder ao objetivo, foram convidados a participar profissionais da psicologia com os cuidados paliativos como campo de atuação. O critério de inclusão adotado foi ter experiência profissional mínima de seis meses. O critério de exclusão adotado foi o participante não estar profissionalmente ativo no momento da pesquisa (ou seja: estar afastado por questões médicas ou de outra natureza).

Para a coleta de dados, foi utilizado um questionário online composto por ficha de caracterização sociodemográfica/ocupacional e questões abertas (1. Qual a maior dificuldade/desafio enfrentado no seu trabalho?; 2. Descreva alguma situação estressora que ocorreu no seu ambiente de trabalho). A utilização do questionário online foi devido à pandemia e a não possibilidade de aplicação de entrevistas presenciais. Para a análise das questões abertas, foi realizada análise de conteúdo temática. Buscou-se classificar as respostas em categorias para auxiliar na compreensão do que está por trás dos discursos, tendo como base o modelo de Bardin (2010). Inicialmente, foi realizada a leitura flutuante dos dados coletados. Após a exploração do material e criação das categorias, foi iniciada a interpretação entrelaçada ao referencial teórico. 
Participaram desse estudo oito psicólogas que atuam em cuidados paliativos na cidade de Salvador/BA, com idades entre 27 e 54 anos. Seis delas afirmaram trabalhar só no contexto de cuidados paliativos e as outras duas atuam, também, em outro contexto hospitalar. O tempo de atuação nesta área variou entre 2 a 20 anos (média de 7 anos). Em respeito ao anonimato das participantes, elas serão referenciadas como participante 1 , participante 2 e assim sucessivamente, até a participante 8 .

Em relação aos requisitos éticos, o presente projeto de pesquisa foi submetido ao Comitê de Ética em Pesquisa da Escola Bahiana de Medicina e Saúde Pública e aprovado em setembro de 2020 sob o número do Parecer: 4.269.364, CAAE:11695019.5.0000.5544 de acordo com a Resolução do Conselho Nacional de Saúde $n^{\circ} \underline{466}$ (2012). A coleta também foi realizada no ano de 2020, nos meses de setembro e outubro, logo após a aprovação do Comitê de Ética.

\section{Resultados e discussão}

As análises das respostas abertas quanto aos desafios enfrentados no cotidiano laboral resultaram na construção de quatro categorias, a saber, "comunicação e atuação com a equipe multiprofissional", "atendimento à pacientes e familiares", "condições de trabalho" e "falta de reconhecimento profissional". Como se tratava de questões abertas era possível uma participante discorrer por diferentes temáticas quando se referia aos desafios enfrentados no trabaIho. Sendo assim, na análise, a sua resposta foi classificada em mais de uma categoria - o que ocorreu com quatro das oito participantes.

\section{Comunicação e atuação com a equipe multiprofissional}

A primeira categoria identificada foi "comunicação e atuação com a equipe multiprofissional", assim denominada por esse conteúdo ter sido identificado com maior frequência, pois surgiu na fala de cinco participantes. Nos dois relatos expostos a seguir é possível observar dificuldades das psicólogas quanto ao trabalho com os demais membros da equipe, que perpassam pela comunicação:
Participante 5: "(...) o médico desconsiderava opinião de equipe multi, inábil no controle de sintomas, projetando na psicologia limitações do próprio nível de conhecimento (...)"

Participante 4: "A maior dificuldade é lidar com profissionais despreparados técnica e emocionalmente"

A interação entre profissionais, disputas de poder entre os saberes e atendimento desrespeitoso ao paciente são algumas das dificuldades apresentadas por profissionais da psicologia hospitalar (Fossi \& Guareschi, 2004; Maturana \& Do Valle, 2014), o que converge com a primeira categoria do presente estudo.

Porém, essa dificuldade de atuar em equipe não é apontada apenas por profissionais da psicologia. Equipes multiprofissionais de cuidados paliativos também destacam dificuldades ao lidar com os colegas e trabalhar em conjunto. Alguns dos pontos ressaltados são: falta de preparo dos profissionais para atuar com a terminalidade e cuidados paliativos, desistência da equipe de lutar pela vida do paciente e dificuldades de relação interpessoal (Cardoso et al., 2013; Alves, Andrade et al., 2015).

Ademais, conflitos na comunicação e atuação com a equipe podem causar desequilíbrio emocional do profissional, o que pode levar à alteração no clima organizacional (Silveira et al., 2014), e que tende a impactar negativamente os colegas de trabalho.

Perboni et al. (2018) entendem que a falta de capacitação na graduação para lidar com a morte, que visa um ensino de abordagem mais biológica e tecnicista pode gerar sofrimentos no exercício futuro da profissão, causando frustrações nos profissionais que não foram educados a lidar com o ser humano e a morte.

Em entrevistas feitas por Braz e Franco (2017) com profissionais de saúde sobre a terminalidade, uma psicóloga destaca a necessidade de haver disponibilidade e envolvimento na atuação profissional, porém com limite. Provavelmente, a identificação desse limite deve ser levada em consideração para estudos posteriores, mas, também, no ensino em cursos de graduação na área de saúde para um melhor atendimento a ser ofertado em pacientes em fim de vida. 


\section{Atendimento à pacientes e familiares}

A segunda categoria foi denominada de "atendimento à pacientes e familiares", agregando conteúdos da relação entre psicóloga-paciente e psicóloga-familiares, que esteve presente nos discursos de três participantes e podem ser percebidos nos relatos a seguir:

\section{Participante 1: "é difícil quando a família não acessa a gravidade da situação/doença"}

Participante 7: "a maior dificuldade é quando não é possível realizar ações que minimizem o sofrimento apresentado"

Esses dados convergem com estudos que trabalharam com equipes paliativistas e que apontaram dificuldades vindas da relação com o paciente ou com a respectiva família, quando se trata do profissional precisar lidar com a morte e/ou comunicar más notícias. Uma possível explicação para tal é a escassez de conteúdo referente a essa temática nos cursos de graduação e, devido a difícil situação que é enfrentar a sua própria finitude, assim como suas frustrações e limitações no papel de profissional de saúde (Cardoso et al., 2013; Alves, Andrade et al., 2015; Silveira et al., 2014).

Lidar com a família é um dos fatores mais difíceis para os profissionais de enfermagem diante do processo de morte e morrer do paciente, podendo vir a gerar sentimentos como angústia e impotência. Já oncologistas pediátricos relatam dificuldade em se comunicar com a família pois, durante o processo de morte e morrer, os pais, principalmente, ficam extremamente irritados com os profissionais de saúde (Perboni et al., 2018).

Em contrapartida, um estudo de Braz e Franco (2017) traz o relato de uma psicóloga que considera a presença do sofrimento da família como algo que é natural e corrente no momento de perda de um ente querido. Essa diferença de perspectiva pode ser explicada pela formação e atuação dos enfermeiros e médicos ser mais pautadas em um tratamento curativo em relação à psicologia, causando uma maior tendência a sentimentos de impotência quando há perdas.

\section{Condições de trabalho}

A terceira categoria se trata de "condições de trabalho" (evidenciada por três participantes) e reúne fatores associados diretamente à organização, e mais especificamente, às políticas e práticas de gestão de pessoas, destacadas nos relatos de duas participantes:

Participante 3: "Enfrento sempre dificuldades relacionadas a um espaço destinado a privacidade para uma escuta familiar que não seja no corredor do hospital"

Participante 6: "Não ter um número suficiente de colegas da mesma classe - sobrecarga de trabalho"

É necessário levar em consideração que temáticas acerca de condições de trabalho devem ser analisadas a partir de um entendimento mais amplo em relação a como a empresa está estruturada, seus valores, poder aquisitivo além de outras variáveis, para que de fato se possa avaliar as condições de trabalho ofertadas.

Outrossim, acrescenta-se que os estudos acerca de condições de trabalho costumam reunir três dimensões: a organização do trabalho (elementos prescritos que expressam as práticas de gestão de pessoas); as condições propriamente ditas (elementos estruturais, desde a infra-estrutura ao suporte organizacional ao trabalhador); e as relações psicossociais (expressam os elementos de interação no trabalho que envolvem relações hierárquicas, entre membros da equipe e entre equipes, e entre os clientes, por exemplo) (Ferreira \& Mendes, 2008).

Os dados observados no presente estudo encontram-se alinhados aos da pesquisa de Lima et al. (2009) com profissionais de saúde, incluindo psicólogas(os), de quatro hospitais nordestinos. Os autores afirmam que, embora não seja possível generalizar os resultados obtidos associados às condições de trabalho, foi evidenciado um perfil de numerosas carências em alguns deles, especialmente no que tange à qualidade dos equipamentos e materiais e ao conforto dos profissionais.

Entretanto, na presente pesquisa, não foram identificadas as precariedades das condições de trabalho como as do estudo de Maturana e Do Valle (2014), realizado com 20 psicólogas(os) hospitalares, que relataram carga horária excessiva, horário do pronto atendimento e mudanças bruscas no ambiente e nas funções do trabalho, as quais são reconhecidas como fonte de estresse. 


\section{Falta de reconhecimento profissional}

A quarta e última categoria foi "falta de reconhecimento profissional", cujo conteúdo surge nas falas das psicólogas de forma literal, sugerindo o quantum de sofrimento que tem sido vivenciado no cotidiano do espaço de trabalho, evidenciado por duas participantes, nas falas destacadas a seguir:

Participante 3: "Me gera estresse a gestão não reconhecer a necessidade de uma psicóloga exclusiva na equipe de cuidado paliativo"

Participante 5: "É difícil lidar com a falta de reconhecimento do conhecimento da profissão"

É possível perceber convergência dessa categoria com o estudo de Fossi e Guareschi (2004), que relata a falta de conhecimento sobre a ajuda que outras especialidades podem dar à equipe e ao indivíduo, como uma dificuldade apontada por psicólogas. Ou seja, o entendimento dos profissionais em relação à prática de cada membro da equipe ainda deixa a desejar, o que pode, inclusive, gerar conflitos relacionais entre os mesmos, prejudicando a qualidade do trabalho e o bem-estar dos profissionais.

Em pesquisa feita por Alves, Andrade et al. (2015), cuidadores profissionais e não profissionais reconheceram a importância do serviço da psicologia, pontuando como os pacientes e familiares se sentem melhor pós-atendimento psicológico, porém dúvidas sobre o papel da psicóloga foram marcadas nos discursos de ambos os grupos de cuidadores. É plausível que a percepção da falta de reconhecimento profissional pode advir da pouca clareza da função do profissional da psicologia nesses ambientes, talvez esteja associada a ainda ser considerado recente em comparação com os outros campos de saber.

É importante destacar a visível relação entre a terceira e última categoria (condições de trabalho e falta de reconhecimento profissional). As dificuldades enfrentadas pelas participantes no que tange as condições de trabalho podem ser compreendidas, além dos fatores organizacionais mencionados, pela falta de uma extensa e firme colocação da psicologia no ambiente hospitalar, quando comparada as outras áreas profissionais.
Primordial ressaltar que, ainda hoje, existem hospitais que não contam com um serviço de psicologia ou até o possuem, porém se trata de uma implementação recente. A inserção da psicóloga nesse ambiente pode vir a contradizer a ordem estabelecida de normatividade da medicina que visa o aspecto orgânico, físico ou biológico (Fossi \& Guareschi, 2004). E, ainda, pelo fato de a Psicologia não ser demandada no primeiro momento quando se busca atendimento hospitalar ou clínico para um enfermo, fatores que podem vir a causar a não prioridade da gestão organizacional sobre esse serviço quando planeja seus investimentos.

Porém, isso está mudando devido à forte evidência de um processo de adoecimento e hospitalização menos traumático e penoso ofertado pela psicologia para pacientes e familiares, em conjunto com a equipe multiprofissional (Alves, Andrade et al., 2015).

\section{Considerações finais}

As categorias dos desafios percebidos pelas profissionais da psicologia no contexto de cuidados paliativos obtiveram maior destaque para "comunicação e atuação com a equipe multiprofissional", seguida de "atendimento à paciente e família" e "condições de trabalho" de forma equivalente, e por último, "falta de reconhecimento profissional".

Diante do presente estudo, se percebe necessário compreender e tratar o trabalhador da saúde como alguém que, para cuidar de maneira satisfatória, também precisa ser cuidado (Cardoso et al., 2013). O presente estudo aponta para as dificuldades enfrentadas pelo profissional da psicologia que atua em cuidados paliativos, o que convoca a refletir sobre os caminhos que devem ser traçados para uma melhora organizacional e com relação à prática deste trabalhador.

O campo de cuidados paliativos segue em ascensão, percebido cada vez mais como relevante e que seguindo a real definição do objetivo dessa área, demanda constantemente de intervenções psicológicas como parte da prática do cuidado integral. Não obstante, o tema da morte, apesar de todos os avanços do movimento paliativista, ainda é considerado um tabu, não sendo discutido de forma crítica e reflexiva nos cursos de graduação em saúde (Alves, Cunha et al., 2019). 
A psicologia, como um campo de saber com vasto estudo sobre luto, pode operar como forte aliado da equipe multiprofissional no melhor preparo para lidar com perdas. $\mathrm{O}$ estresse vivido por pacientes $\mathrm{e}$ familiares nos processos de adoecimento, hospitalização e/ou morte é considerado por alguns profissionais como maior desafio, sendo necessário um maior preparo para lidar com essas situações, assim como suporte e lugares de fala, para que possam expor as suas emoções e dificuldades, livremente.

Contudo, em comparação aos demais profissionais de saúde, os da psicologia se mostram mais preparados para lidar com essa temática, mas ainda despreparados no que tange a inserção na equipe multiprofissional, comunicação e atuação com a mesma. Dar visibilidade a essas lacunas é essencial para averiguação das razões referentes às dificuldades visando a redução das tensões ocupacionais, o que favorece todos os atores envolvidos no processo, especialmente o paciente.

Ademais, é muito importante que os cursos de graduação em saúde, especialmente os de psicologia, ampliem a formação do estudante quanto aos estudos sobre a morte e seus significados em nossa sociedade, trabalhando em componentes curriculares teóricos e práticos à referida temática, ou ainda ofertando atividades extracurriculares. Mesmo considerando a evolução da psicologia no campo hospitalar, além do fato de algumas áreas já estarem "enturmadas" com o serviço psicológico, $33 \%$ dos serviços de cuidados paliativos, no Brasil, não possuem psicólogas com dedicação exclusiva e 53\% têm, apenas, um profissional da área, o que demonstra escassez de profissionais da psicologia nesse contexto (Santos et al., 2020).

Como limitações do estudo, pode-se destacar o contexto de pandemia da Covid-19 em que a coleta foi realizada, o que pode ter gerado um viés nos dados obtidos, já que os profissionais se encontravam em uma situação atípica e de sobrecarga laboral. O outro fator se refere ao fato da pesquisa ter sido realizada de forma online (via formulário), o que não permite aprofundamento e esclarecimento das respostas ou inserção de novas perguntas de acordo com o caminhar da entrevista. Também não foram abordadas características como vínculo de trabalho (setor público ou privado) e perfil dos respectivos pacientes das psicólogas. Assim sendo, sugere-se a realização de novos estudos para investigar mais a fundo os desafios enfrentados pelos profissionais da psicologia como, também, pelos outros integrantes da equipe multiprofissional.

\section{Contribuições das autoras}

Edington RN participou da concepção, análise de dados e produção textual. Aguiar CVN e Silva EEC participaram da orientação, análise de dados, produção textual e correção do estudo.

\section{Conflitos de interesses}

Nenhum conflito financeiro, legal ou político envolvendo terceiros (governo, empresas e fundações privadas, etc.) foi declarado para nenhum aspecto do trabalho submetido (incluindo, mas não se limitando a subvenções e financiamentos, participação em conselho consultivo, desenho de estudo, preparação de manuscrito, análise estatística, etc.).

\section{Referências}

Academia Nacional de Cuidados Paliativos. (s.d.). O que são cuidados paliativos? https://paliativo.org.br/cuidadospaliativos/o-que-sao

Alves, R. F., Andrade, S. F. O., Melo, M. O., Cavalcante, K. B., \& Angelim, R. M. (2015). Cuidados paliativos: desafios para cuidadores e profissionais de saúde. Fractal: revista de psicologia, 27(2), 165-176. https://doi.org/10.1590/19840292/943

Alves, R. S. F., Cunha, E. C. N., Santos, G. C., \& Melo, M. O. (2019). Cuidados paliativos: alternativa para o cuidado essencial no fim da vida. Psicologia: Ciência e Profissão, 39, 1-15. https://doi.org/10.1590/1982-3703003185734

Bardin, L. (2010). Análise de conteúdo (4a ed.). Edições70.

Braz, M. S., \& Franco, M. H. P. (2017). Profissionais paliativistas e suas contribuições na prevenção de luto complicado. Psicologia: Ciência e Profissão, 37(1), 90-105. https://doi. org/10.1590/1982-3703001702016

Cardoso, D. H., Muniz, R. M., Schwartz, E., \& Arrireira, I. C. O. (2013). Cuidados paliativos na assistência hospitalar: a vivência de uma equipe multiprofissional. Texto \& Contexto Enfermagem, 22(4), 1134-41. https://doi.org/10.1590/ S0104-07072013000400032

Carvalho, R. T., \& Parsons, H. A. (Orgs.). (2012). Manual de cuidados paliativos ( $2^{\mathrm{a}}$ ed.). ANCP. http://biblioteca.cofen.gov.br/wpcontent/uploads/2017/05/Manual-de-cuidados-paliativosANCP.pdf 
Ferreira, M. C., \& Mendes, A. M. B. (2008). Contexto de trabalho. In M. M. M. Siqueira, (Org.). Medidas do comportamento organizacional: ferramentas de diagnóstico e de gestão. Artmed.

Fossi, L. B., \& Guareschi, N. M. F. (2004). A psicologia hospitalar e as equipes multidisciplinares. Revista da SBPH, 7(1), 29-43. http://pepsic.bvsalud.org/scielo.php?script=sci arttext\&pid=S1516-08582004000100004

Gomes, A. L. Z., \& Othero, M. B. (2016). Cuidados paliativos. Estudos avançados, 30(88), 155-166. https://www.revistas. usp.br/eav/article/view/124275

Hermes, H. R., \& Lamarca, I. C. A. (2013). Cuidados paliativos: uma abordagem a partir das categorias profissionais de saúde. Ciência \& Saúde Coletiva, 18(9), 2577-2588. https://doi. org/10.1590/S1413-81232013000900012

Lei n. 4.119, de 27 de agosto de 1962. (1962). Dispõe sobre os cursos de formação em psicologia e regulamenta a profissão de psicólogo. http://www.planalto.gov.br/ ccivil_03/leis/1950-1969/L4119.htm

Lei n. 5.766, de 20 de dezembro de 1971. (1971). Cria o Conselho Federal e os Conselhos Regionais de Psicologia e dá outras providências. http://www.planalto.gov.br/ccivil_03/ leis/15766.htm

Lima Júnior, J., Alchieri, J. C., \& Maia, E. M. C. (2009). Avaliação das condições de trabalho em hospitais de Natal, Rio Grande do Norte, Brasil. Revista da Escola de Enfermagem USP, 43(3), 670-676. https://doi.org/10.1590/S0080$\underline{62342009000300024}$

Maturana, A. P. P. M., \& Do Valle, T. G. M. (2014). Estratégias de enfrentamento e situações estressoras de profissionais no ambiente hospitalar. Psicologia Hospitalar, 12(2), 2-23. http://pepsic.bvsalud.org/scielo.php?script=sci arttext\&pid=S1677-74092014000200002

Organização Mundial de Saúde. (2002). Definition of palliative care [Definição de tratamento paliativo]. OMS. https://www. who.int/westernpacific/health-topics/palliative-care

Perboni, J. S., Zilli, F., \& Oliveira, S. G. (2018). Profissionais de saúde e o processo de morte e morrer dos pacientes: uma revisão integrativa. Persona y bioética, 22(2), 288-302. http://www.scielo.org.co/pdf/pebi/v22n2/0123-3122pebi-22-02-00288.pdf

Resolução n. 013, de 14 de dezembro de 2007. (2007). Institui a Consolidação das Resoluções relativas ao Título Profissional de Especialista em Psicologia e dispõe sobre normas e procedimentos para seu registro. http://site. cfp.org.br/wp-content/uploads/2008/08/Resolucao_CFP_ nx_013-2007.pdf
Resolução n. 014, de 20 de dezembro de 2000. (2000). Institui o título profissional de Especialista em Psicologia e dispõe sobre normas e procedimentos para seu registro. https://site.cfp.org.br/wp-content/uploads/2006/01/ resolucao2000_14.pdf

Resolução n. 02, de 10 de março de 2001. (2000). Altera e regulamenta a Resolução CFP no 014/00 que institui o título profissional de especialista em psicologia e o respectivo registro nos Conselhos Regionais. https://site. cfp.org.br/wp-content/uploads/2006/01/resolucao2001__. pdf

Resolução n. 1.973, de 1 de agosto de 2011. (2011). Dispõe sobre a nova redação do Anexo II da Resolução CFM n $1.845 / 2008$, que celebra o convênio de reconhecimento de especialidades médicas firmado entre o Conselho Federal de Medicina (CFM), a Associação Médica Brasileira (AMB) e a Comissão Nacional de Residência Médica (CNRM). https://www3.semesp.org.br/portal/pdfs/ juridico2011/Resolucoes/Res_CFM_1973_14.07.pdf

Resolução n. 466, de 12 de dezembro de 2012. (2012). Incorpora, sob a ótica do indivíduo e das coletividades, referenciais da bioética, tais como, autonomia, não maleficência, beneficência, justiça e equidade, dentre outros, e visa a assegurar os direitos e deveres que dizem respeito aos participantes da pesquisa, à comunidade científica e ao Estado. http://conselho.saude.gov.br/resolucoes/2012/ Res0466.pdf

Santos, A. F. J., Ferreira, E. A. L., \& Guirro, U. B. P (Orgs.). (2020). Atlas de Cuidados Paliativos no Brasil 2019. Academia Nacional de Cuidados Paliativos. https://api-wordpress. paliativo.org.br/wp-content/uploads/2020/05/ ATLAS_2019_final_compressed.pdf

Santos, T. F., \& Pintarelli, V. L. (2019). Educação para o Processo do Morrer e da Morte pelos Estudantes de Medicina e Médicos Residentes. Revista Brasileira de Educação Médica, 43(2), 5-14. https://doi.org/10.1590/1981$52712015 v 43 n 2 R B 20180058$

Silva, R. P., Barbosa, S. C., Silva, S. S., \& Patrício, D. F. (2015). Burnout e estratégias de enfrentamento em profissionais de enfermagem. Arquivos Brasileiros de Psicologia, 67(1), 130-145. http://pepsic.bvsalud.org/scielo.php?script=sci arttext\&pid=S1809-52672015000100010

Silveira, M. H., Ciampone, M. H. T., \& Gutierrez, B. A. O. (2014). Percepção da equipe multiprofissional sobre cuidados paliativos. Revista Brasileira de Geriatria e Gerontologia, 17(1), 7-16. https://doi.org/10.1590/S1809. 98232014000100002 